ACCEPTED MANUSCRIPT

\title{
Introducing a biomimetic coating for graphene neuroelectronics: toward in-vivo applications
}

To cite this article before publication: Antoine Bourrier et al 2019 Biomed. Phys. Eng. Express in press https://doi.org/10.1088/2057-1976/ab42d6

\section{Manuscript version: Accepted Manuscript}

Accepted Manuscript is "the version of the article accepted for publication including all changes made as a result of the peer review process, and which may also include the addition to the article by IOP Publishing of a header, an article ID, a cover sheet and/or an 'Accepted

Manuscript' watermark, but excluding any other editing, typesetting or other changes made by IOP Publishing and/or its licensors"

This Accepted Manuscript is @ 2019 IOP Publishing Ltd.

During the embargo period (the 12 month period from the publication of the Version of Record of this article), the Accepted Manuscript is fully protected by copyright and cannot be reused or reposted elsewhere.

As the Version of Record of this article is going to be / has been published on a subscription basis, this Accepted Manuscript is available for reuse under a CC BY-NC-ND 3.0 licence after the 12 month embargo period.

After the embargo period, everyone is permitted to use copy and redistribute this article for non-commercial purposes only, provided that they adhere to all the terms of the licence https://creativecommons.org/licences/by-nc-nd/3.0

Although reasonable endeavours have been taken to obtain all necessary permissions from third parties to include their copyrighted content within this article, their full citation and copyright line may not be present in this Accepted Manuscript version. Before using any content from this article, please refer to the Version of Record on IOPscience once published for full citation and copyright details, as permissions will likely be required. All third party content is fully copyright protected, unless specifically stated otherwise in the figure caption in the Version of Record.

View the article online for updates and enhancements. 


\section{Introducing a biomimetic coating for graphene}

\section{neuroelectronics: toward in-vivo applications}

Antoine Bourrier ${ }^{a}$, Anna Szarpak-Jankowska ${ }^{b}$, Farida Veliev $^{a}$, Renato Olarte-Hernandez ${ }^{a}$,

Polina Shkorbatova ${ }^{c}$, Marco Bonizzato $^{c}$, Elodie Rey ${ }^{c}$, Quentin Barraud ${ }^{c}$, Anne Briançon-

Marjollet, ${ }^{d}$ Rachel Auzely ${ }^{b}$, Gregoire Courtine ${ }^{c}$ Vincent Bouchiat ${ }^{a}$, and Cécile Delacour ${ }^{*}, a$

anstitut Néel, CNRS \& Université Grenoble Alpes, 38042 Grenoble, France.

bUniversity Grenoble Alpes, CERMAV-CNRS, 38000 Grenoble, France.

${ }^{\mathrm{c} C e n t e r}$ for Neuroprosthetics and Brain-Mind Institute, School of Life Sciences, Swiss Federal Institute of Technology (EPFL), Campus Biotech CH-1202 Geneva, Switzerland.

${ }^{\mathrm{d}}$ Grenoble Alpes, HP2 Laboratory, Institut National de la Santé et de la Recherche Médicale U1042, Grenoble, France

KEYWORDS. Biomaterial, graphene, surface functionalization, hyaluronic acid, bioelectronics, intracortical implant, neurons 
ABSTRACT. Electronic micro and nano-devices are suitable tools to monitor the activity of many individual neurons over mesoscale networks. However the inorganic materials currently used in microelectronics are barely accepted by neural cells and tissues, thus limiting both the sensor lifetime and efficiency. In particular, penetrating intracortical probes face high failure rate because of a wide immune response of cells and tissues. This adverse reaction called gliosis leads to the rejection of the implanted probe after few weeks and prevent long-lasting recordings of cortical neurons. Such acceptance issue impedes the realization of many neuro-rehabilitation projects. To overcome this, graphene and related carbon-based materials have attracted a lot of interest regarding their positive impact on the adhesion and regeneration of neurons, and their ability to provide high-sensitive electronic devices, such as graphene field effect transistor (G-FET). Such devices can also be implemented on numerous suitable substrates including soft substrates to match the mechanical compliance of cells and tissues, improving further the biocompatibility of the implants. Thus, using graphene as a coating and sensing device material could significantly enhance the acceptance of intracortical probes. However, such a thin monolayer of carbon atoms could be teared off during manipulation and insertion within the brain, and could also display degradation over time. In this work, we have investigated the ability to protect graphene with a natural, biocompatible and degradable polymeric film derivated from hyaluronic acid (HA). We demonstrate that HA-based coatings can be deposited over a wide range of substrates, including intracortical probes and graphene FET arrays without altering the underlying device material, its biocompatibility and sensitivity. Moreover, we show that this coating can be monitored in-situ by quantifying the number of deposited charges with the G-FET arrays. The reported graphene functionalization offers promising alternatives for improving the acceptance of various neural interfaces. 
INTRODUCTION. Since the early 80 s, the emergence of the microelectronic industry and devices miniaturization has enabled the development of brain implants carrying microelectrode arrays capable of detecting single spikes from multiple individual neurons over days and months. ${ }^{1,2}$ These neurotechnologies led to pivotal discoveries in fundamental brain research and supported remarkable breakthroughs in neuroprosthetic medicine. ${ }^{3,4}$ However, the poor biocompatibility of intracortical probes still prevents chronic sustainable recordings and thus restricts the spectrum of applications that could be addressed. As of today, the immune response of neural tissues and the low adhesion of neurons onto the electrode material limit the efficiency and time stability of current neural interfaces. ${ }^{5}$

While the electrical activity of neuron assemblies can/be recorded remotely from the surface of neural tissues (with electroencephalography EEG or EcoG for instance), the detection of single spike requires a near field detection, i.e. approaching the sensing materials in close contact with the targeted neurons. This implies the insertion of electrodes within the brain. Consequently, the biocompatibility, the mechanical compliance, the surface charge and topography, and the chemical stability of implanted neural interfaces have critical impact on the performances of the sensors carried on the neural probe. ${ }^{6,7}$

Currently, the insertion of those devices through cortical brain areas leads to strong tissue response (trauma) that renders the coupling with the surrounding cells ineffective. In particular, a thick layer (about $100-200 \mu \mathrm{m}$ ) of microglia and reactive astrocyte has been shown to surround the implanted devices after few weeks, and to prevent an intimate contact with the targeted neurons. ${ }^{8}$ Consequently, this growing layer of glial cells partially shields the signal emitted by neurons and contributes to reduce the sensor signal-to-noise ratio over time, eventually leading to a high-rate of deyice failure. In addition, micro-movements of the rigid implant could also trigger an 
inflammatory response that further contributes to increase the distance between the cells and the sensors. Moreover, the presence of reactive oxygen species and free imbalanced charge can also damage the implanted electronics, both the sensors and the passivation layer, and further reduce the sensitivity, efficiency and time reliability of the devices. ${ }^{9}$

Over the past decade, graphene have attracted numerous interests to alleviate some of the issues faced by neuroelectronics. Indeed, it provides a unique combination of exceptional properties suitable for bio and neuroelectronics, such as its exceptional neural affinity, ${ }^{10-12}$ chemical inertness, bendability ${ }^{13}$ (low bending rigidity while having a very strong tensile strength), anti-corrosive and anti-oxidative properties, ${ }^{14,15}$ as well as its high electrical conductivity and charge carriers mobility. Indeed its sensitivity and frequency range of operation exceed those of conventional semiconductors. Additionally, the low-cost and industrial scale production of polycrystalline monolayer obtained by chemical vapor deposition (CVD) growth method, further promotes graphene as an ideal material for future neural probes. ${ }^{16,17}$ Using monolayer graphene has other advantages, regarding the low quantity of inserted electrodes material that reduces the toxicity of the implanted material. Also, monitoring the monolayer conductance provides an accurate control in-situ of its structural integrity.

However, owing to its ultimately small thickness, the possible degradation of graphene $\mathrm{e}^{18-20}$ together with the mechanical frictions with surrounding cells and tissues could easily lead to device failures. To overcome this drawback, we have investigated the ability to functionalize intracortical probes and graphene monolayers with a biocompatible, soft and bioresorbable polymeric film. Such coating could protect the sensors during their insertion, and possibly favor the healing process around the implants by improving the chemical and mechanical compliance with the soft neural tissues. ${ }^{21}$ 
In recent years, hyaluronic acid HA has been widely used as a biomaterial for different clinical applications. $^{22}$ This anionic polysaccharide is a major component of the extracellular matrix of connective tissues. The interactions of HA with cellular HA-binding proteins are important for cell adhesion, proliferation, wound healing and the HA binding sites might also provide the possibility for selective cell adhesion. ${ }^{23}$ In addition, HA monolayer could be used as basal layer for further hydrogel functionalization, to soften the interface and improve the mechanical compliance with neural cells, in order to promote neurite outgrowth ${ }^{24}$ or to provide new functionalities such as growth factors delivery for nerve regeneration. ${ }^{25}$ In addition with these many advantages, both the thickness and biodegradation rate of HA coatings can be controlled in order to maintain a close and tiny contact with cells for an efficient recording. Until now, some examples of HA-coated electrodes have been tested in-vivo ${ }^{26}$ or in-vitro with platinium, iridium microwires and silicon microelectrodes. ${ }^{27,28}$ The challenge here lies in the maintenance of electrodes performances after the coating process.

Here, we report on the synthesis of HA-based films and show the ability to implement it onto intracortical probe and on monolayer graphene without altering both the material and device features, in term of biocompatibility, detection efficiency and sensitivity.

\section{MATERIALS AND METHODS}

Biopolymer coating. For the HA/PLL multilayers deposition, the samples are exposed to a solution of hyaluronic acid (HA) $\left(\mathrm{M}_{\mathrm{w}}=200 \mathrm{~kg} / \mathrm{mol}\right.$ MEDIPOL Distribution, dissolved at $2 \mathrm{mg} / \mathrm{ml}$ in $0.15 \mathrm{M} \mathrm{NaCl}$ solution adjusted at $\mathrm{pH} 6.5$ ) for $10 \mathrm{~min}$, then rinsed in three successive baths of $0.01 \mathrm{M} \mathrm{NaCl}$ solution during $5 \mathrm{~min}$. They are then exposed to poly-L-lysine (PLL) $(2 \mathrm{mg} / \mathrm{mL}$, Sigma-Aldrich), and further rinsed in $0.01 \mathrm{M} \mathrm{NaCl}$ solution ( $\mathrm{pH} 6.5$ ). The procedure was repeated 
several times to obtain the desired number of HA/PLL bilayers (from $n=1$ to 5). Alkylamino hydrazide derivatives of hyaluronic acid ( $\alpha-\mathrm{HA})$ were synthesized from HA as previously described, ${ }^{29}$ and allowed to enhance the adhesion of the hyaluronic acid on the hydrophobic graphene samples. Alkyl chains having ten carbon atoms $(\mathrm{C} 10)$ were grafted on HA backbone with a substitution degree (DS, number of substituents per repeating disaccharide unit of HA) of 0.1 or 0.2. The water used in all experiments was purified by a Millipore Milli-Q Plus purification systems, with a resistivity of $18.2 \mathrm{M} \Omega \cdot \mathrm{cm}$. Samples are kept in stable $\mathrm{pH}$ solution $(0.01 \mathrm{M} \mathrm{NaCl})$ until their used to preserve the polymers integrity. Shortly before cells seeding, the buffer solution is rinsed and replaced by the attachment culture medium. Finally, primary hippocampal neurons (extracted from mousse embryo E16, see methods) are introduced in the solution.

Pure neuron culture and immunofluorescent staining. Neurons were extracted from the hippocampus of mouse embryos (E16.5 pregnant mice from Envigo, killed by cervical elongation), dissociated and seeded with a density of 150000 cells $/ \mathrm{cm}^{2}$ in attachment medium (MEM supplemented with $10 \%$ foetal bovin serum, $1 \%$ glutamine and $0.05 \%$ peniciline/streptomycine, Gibco). Neurons were then incubated at $37^{\circ} \mathrm{C}$ and $5 \% \mathrm{CO}_{2}$, and the attachment medium was replaced few hours later by glial conditioned culture medium, supplemented with $\mathrm{AraC}(1 \mathrm{mM})$ to suppress the glial proliferation. The glial conditioned medium is previously obtained by culturing glial cells from the cortex in attachment medium until confluency. The cells were then cultivated with serum free Neurobasal medium (Invitrogen) and the glial conditioned medium was collected after $48 \mathrm{~h}$ for subsequent use on neuron cultures.

For the immunofluorescent staining, neurons were fixed in $4 \%$ formaldehyde (prepared from fresh paraformaldehyde) (10 min), permeabilized in PBS-0.25\% Triton-X100, blocked in PBS-2\% bovine serum albumin and finally immunostained with the anti-Synapsin (1:500, Millipore), anti- 
Tau (1:300, Millipore) and anti-YL1/2 (1:1000, BioRad) primary antibodies, and DAPI(1:1000, Sigma-Aldrich) for labeling the synaptic vesicles, the axon, the cytoskeleton and the nucleus respectively. Immunoreactions were visualized with appropriate secondary antibodies labeled with Alexa fluor ${ }^{\circledR} 488$ or 594 or 647 (1:500, Life Technologies). Immunofluorescence images were collected using automatic Zeiss Axio-Imager M2 microscope, and the image processing was performed with ImageJ..$^{30}$

Statistics. For cell density assessment, somas are counted using Image-J particles analysis plugin after picture conversion in 16 bits and threshold definition to reject the background noise. Soma size has first been measured manually on a dozen of neurons per picture in order to define the average soma area to detect. For groups of cells, each soma cannot be discriminated, thus the number is estimated by dividing the total area of clusters by the average soma size, and then verified with manual counting. The covering rate for neurite and axon have been extracted by adjusting the threshold and preserving a constant brightness level for each fluorescent antibodies. Defocus - inducing less brightness and larger covering surfaces - is taking into account by keeping the same threshold level of brightness between all pictures. For synapses counting, a high threshold was used to detect bright puncta around the soma, and a low threshold was used to detect puncta along neurites.

In-vivo assays. Six Adult female Lewis rats (200-225 grams, N.23, Charles River, UK) were housed individually on $12 \mathrm{~h}$ light/dark cycle with access to food and water ad libitum. Before surgery, animals were first habituated to human presence and manipulation during 2 weeks. Stereotaxic fixation was performed after administration of $0.1 \mathrm{ml}$ of Dorbene anaesthesia and surgeries were performed under inhalant anaesthesia of $2 \%$ diluted isofluorane in $2 \mathrm{~L} / \mathrm{min}$ oxygen. Briefly, we used 6 arrays with 32 recording channels: 3 arrays of coated microelectrodes, and 3 
bare (uncoated) arrays for the control, leading to 96 independent microelectrodes tested for each conditions (182 electrodes in total) on 6 independent rodents. Each recording channel is a thin (100 $\mu \mathrm{m}$ ) tungsten wire encapsulated by polyimide (from Tucker-Davis-Technologies, USA). The arrays were inserted into layer $\mathrm{V}$ of the leg region of the right motor cortex, which we previously identified anatomically and electrophysiologically. ${ }^{31}$ Signal amplification and recording were made with the Tucker Davis Technologies PZ2 preamp and RZ2 amplifier at the sampling frequency of $25 \mathrm{kHz}$. Spike sorting is processed in real-time by using TdTC OpenEx (C commercial software and spikes-of-interest were stored when spike occurrence was matching with the walking status. We labeled the corresponding sensors among the 32 recording sites of each implant. The experience was repeated once a week by in-cage free-movement recordings for all sensors and compared to previous results. All procedures and surgeries were approved by the Veterinary Office of the canton of Vaud and the Veterinary Office of the Canton of Geneva (Switzerland).

Histology. At the end of the experimental procedures, rats were perfused with Ringer's solution containing $100000 \mathrm{IU} / \mathrm{L}$ heparin and $0.25 \% \mathrm{NaNO}_{2}$ followed by $4 \%$ phosphate buffered paraformaldehyde, $\mathrm{pH} 7.4$ containing $5 \%$ sucrose. The brains were dissected, post-fixed overnight, and transferred to $30 \%$ phosphate buffered sucrose for cryoprotection. After 4 days, the tissue was embedded and the brains sectioned in a cryostat (Leica, Germany) at a $40 \mu \mathrm{m}$ thickness. Astrocyte and microglial reactivity were revealed by performing immunohistological stainings against glial fibrillary acidic protein (GFAP) and ionized calcium binding adapter molecule 1 (Iba1), respectively. Briefly, the brain sections were incubated overnight with the anti-Iba1 (1:1000, Abcam) or anti-GFAP (1:1000, Dako) primary antibodies. Immunoreactions were visualized with appropriate secondary antibodies labeled with Alexa fluor® 488 or 555 (Life Technologies). Fluorescence counterstaining of Nissl substance was done using neurotrace 
Graphene substrates. Graphene monolayers have been grown by pulsed CVD technique ${ }^{33}$ on copper foils ( $25 \mu \mathrm{m}$ thick, $99.8 \%$ purity, Alfa-Aesar), using time-exposure of $\mathrm{CH}_{4}(2 \mathrm{sccm} 10 \mathrm{~s}$, then 60s off, repeated during an hour) flow in diluted $\mathrm{H}_{2}$ atmosphere (1000 sccm, $25 \mathrm{mbar}$ pressure) to prevent the development of multilayer patches. Prior the growth, the $\mathrm{Cu}$ foil has been cleaned in acetone and further annealed in diluted $\mathrm{H}_{2}$ atmosphere ( $10 \%$ in $\left.\mathrm{Ar}\right)$ at $1000^{\circ} \mathrm{C}$ for $2 \mathrm{~h}$. Then, $4 \times 4 \mathrm{~mm}$ graphene pieces were transferred on $12 \mathrm{~mm}$ wide glass coverslip (Marienfeld) by polymer assisted wet transfer technique. The $\mathrm{Cu}$ foil, covered by PMMA resist on the graphene side, is wet-etched in ammonium persulfate solution $(0.1 \mathrm{~g} / \mathrm{ml}, 2 \mathrm{~h})$. Once the Cu-etching is complete, the floating graphene-PMMA bilayer is rinsed with several baths of DI water to remove all traces of the Cu-etchant solution, and then fished with a cleaned and hydrophilic substrate. The substrate coated with graphene-PMMA is dried at room temperature and PMMA is removed in an acetone bath (overnight, room temperature). The transferred graphene sheets are then characterized using atomic force microscope and Raman microspectroscopy. 
Field effect transistor array. As previously reported, ${ }^{16}$ the graphene sheet is etched to define 20 $\times 10 \mu \mathrm{m}^{2}$ wide and long transistor channels, by using photoresist mask and oxygen plasma etching of the unprotected graphene parts. Then, this first photoresist mask is removed, and a second photoresist mask defines the negative pattern of the contact leads. A metallic thin film (Ti/Au) is then evaporated in vacuum, and the resist is removed in an acetone bath. Finally, a biocompatible SU-8 photo-sensitive polymer is used to insulate the contact leads from the top liquid and to expose the graphene transistors channel only (both DI water, PBS and cell medium culture were tested for the liquid gating experiment showing no significant difference on the result).

\section{RESULTS AND DISCUSSION}

Impact of hyaluronic acid on neurons adhesion and neuritogenesis in-vitro. Isolated hippocampal neurons have been selected to study the behaviour of nerve cells on HA films. Because they are highly sensitive to their microenvironment (topography, chemistry, surface charges, rigidity of the substrate and surrounding medium), they are suitable candidates to evaluate the affinity of a micro-engineered interface. Their affinity to the environment is assessed by measuring their response in term of cell adhesion and neuritogenesis. For that purpose, neurons have been cultured on conventional glass coverslips coated with hyaluronic acid/poly-L-lysine (HA/PLL) multilayer films obtained by layer-by-layer LbL method, ${ }^{34}$ and on PLL single layers covered substrates, as control samples (figure 1).

When neurons were seeded on a single layer of negatively charged HA (glass-coated) substrate, we notice that they barely attached (figure 4c). This is in stark contrast to the behaviour of neurons seeded on single layer of PLL, or on multilayer films obtained by combination of HA and PLL, that promote adhesion and neurites regrowth (figure 1 and 2). 
While the number of attached soma are almost the same on the PLL and HA/PLL samples, the area covered by neurites is twice higher on the multilayer films than on single PLL layer after 5 in culture (figures 1b). Axonal differentiation and synaptogenesis are also significantly enhanced on the HA/PLL films, regarding the axon length and synapse density, respectively. Similar results were obtained for both studied HA/PLL films with PLL or HA as an outer Tayer, (HA/PLL)5/5 and (HA/PLL)5/4, respectively, with slightly higher values for films with PLL on the top. The positive impact of the HA/PLL film on neurons density and neurite spreading is also observed on mature network (figure 2 and figure S1,S2). The neurons density and the surface covered by neurites are still enhanced on the bilayers after 14 days (figure S1), indicating that HA/PLL films support longterm cell regeneration and survival. Moreover, neurons cultured on five (HA/PLL) bilayers deposited on graphene monolayer appears further developed (figure S1), combining both positive impacts of the monolayer graphene and the (HA/PLL) bilayers.

The lack of adhesion on negatively-charged HA monolayer and at the opposite the successful neural development on PLL-containing films confirm that the presence of positive charges impacts the adhesion and spreading rates of cultured neurons. Indeed, the negatively charged cell membrane tends to adhere on positively charged surfaces by electrostatic interaction, as already reported within numerous studies including on nanostructures, ${ }^{35}$ carbon nanotubes ${ }^{36}$ and graphene. ${ }^{12}$ Similarly, Min $\mathrm{Hu}$ et al. showed earlier ${ }^{37}$ that cross-linked HA strands does not permit attachment of Schwann cells Adhesion and proliferation were possible only after coating the surface with PLL. Also, Z. Yue et al. ${ }^{38}$ showed that HA alone was inefficient for cellular growth, consequently an additional modification of a surface with collagen was necessary to improve cell growth and neural differentiation of PC12. 
In addition to the presence of positive charges in the adhesion films, other material features seem to support neurons regrowth on the HA/PLL multilayers, as the adhesion and neurites outgrowth is higher on those samples than on PLL monolayer. Beside the type of biomaterial used to mimic the extracellular matrix ECM, the cells adhesion and outgrowth could be affected by many different cues such as growth factors, stiffness, topography and cell-to-cell contacts. ${ }^{39}$ We have shown previously that multilayer films of five bilayers are also thicker $(>80 \mathrm{~nm})$ than a single monolayer of polymer (< $10 \mathrm{~nm}$ thick), ${ }^{34}$ and more soft than glass coverslip, with a Young's modulus of few $\mathrm{kPa}$ and hundreds of GPa respectively ${ }^{40-41}$ Consequently, the mechanical compliance with neurons might be enhanced on the thick multilayers, and this could improve the neuritogenesis as it was observed in our case on HA/PLL multilayers. Similar enhancement regarding the neurites spreading was also observed when using polydimethylsiloxane (PDMS)coated glass substrates instead bare glass coverslips (fígure S3). The Young's modulus of such elastomers being one order of magnitude lower than for glass (1-10 MPa and $10 \mathrm{GPa}$ respectively), ${ }^{42-44}$ it should also soften the surface sensed by neurons. Although it remains higher than for the nervous system (in the kPa range). Recent works also showed that by regulating the HA hydrogels stiffness and cell-adhesive RGD ligand density, one can promote the neurite outgrowth within 3D matrix without using PLL. ${ }^{24}$ The topography of multilayer films could be another contributing factor, as it is an additional advantage for cell migration and neurite spreading. The presence of biomimetic niches within the HA/PLL film have indeed been shown to promote stem cells adhesion and maturation. ${ }^{45}$

These results show that combining HA and PLL polyelectrolytes, together with the lowered rigidity offered by multilayer film, positively impact the adhesion and spreading rates of primary hippocampal neurons in culture. The LbL method used for the film formation is an easy-to-use 
method which can be easily applied for coating different surfaces, including three-dimensional (3D) intracortical neural probes.

Impact of HA films on intracortical probe biocompatibility. We have investigated the biocompatibility of the HA/PLL coating in-vivo, by depositing the developed multilayer films on conventional intracortical electrodes (tungsten wires, Utah Array, from Tucker Davis technologies (C). These probes were inserted within deep cortical layers to monitor neural assemblies that command hindlimb motion. During 7 weeks of recordings, we have followed the efficiency of the coated electrodes to detect single spike associated to the walking status and their reliability in time. We have also compared the glial cell proliferation around the functionalized (active) and bare (control) probes. These in-vivo investigations were performed as double-blind experiments.

In particular, we have functionalized 32 independent próbes with five bilayers of HA/PLL (figure 3a), deposited by LbL method as for the previous in-vitro assays. The coated probes were compared with 32 independent uncoated probes (controls), in term of device and immune responses. The experiments were performed on two groups of independent rodents: one group of three control rats (uncoated probes), and a second group of three active rats (with the coated probes). The targeted axonal projections of motor neurons is located in the cortical layers $\mathrm{V}$ involved in the hindlimb locomotion command. ${ }^{46}$ Freely-moving recordings were performed once a week on each implanted rodents (supplementary video 1). After five weeks, recordings were performed during walking controlled motion on a treadmill, and compared with the muscle kinetics and locomotion behaviours. The detected neural spikes were then extracted from the voltage timetraces of the 32 probes acquired during rodent walks. Spikes were further analyzed in term of shape, amplitude and frequency to track motor neurons spiking activity. The electrodes which 
successfully detect single spiking events from motor neurons associated to walking status were followed along the 7 weeks.

Clear signals with high signal to noise ratio $(\mathrm{S} / \mathrm{N}>3)$ were extracted from the functionalized probes for all the three experiments (figure $3 b-c$ ). At the opposite, only a single experiment performed with uncoated control probes showed similar high-quality signals (figure S4). We checked that the single spikes detected with the functionalized probes were regular and synchronized with trunk and legs movements, demonstrating an efficient interfacing of motor neurons with the coated probes. Specially, we have compared the number of electrodes able to detect single spikes from individual motor neurons (spikes duration and amplitude being about $1 \mathrm{~ms}$ and $100-200 \mu \mathrm{V}$ as shown figure $3 \mathrm{c}$ and $3 \mathrm{c}$, and associated to the walking status with and without the multilayers coating. Interestingly, the total number of operating electrodes appears to be slightly improved for the probes coated with the polymeric film, being almost twice higher than the number of uncoated (control) probes after the glial scaring process, i.e. 2 weeks typically (figure 3d).

We notice that the disruption of the blood vessels during the implantation surgery could be a source of variability, because of the large amount of blood macrophages and endothelial cells carried inside by the implant could further increase the gliosis and eventually the detection failure. Here, the probes coated with HA/PLL bilayers were still able to sense and discriminate single units (i.e. motor neuron spike) during the highest bleeding level (figures S4, S5 and S6), while no signal was recorded with the control (uncoated) probes at lower bleeding level which further demonstrates the positive impact of the multilayers. 
At the end of the recording procedure, the brains were dissected, post-fixed overnight, and transferred into $30 \%$ phosphate buffered sucrose for cryoprotection. Thin brains slices ( $40 \mu \mathrm{m}$ thick) were stained with immuno-fluorescent proteins for labelling the neurons and the glial cells at the implantation sites. Figure 3e shows representative immuno-fluorescent micrographs of the whole coronal brain sections stained with GFAP or Iba1 and Dapi or Nissl, illustrating the proliferation of astrocytes or microglial cells (red), and the population of neurons (blue), respectively. As expected, those commercial probes caused a significant immuno-response (figure 3f). For some probes, the bleeding level was high and could have an impact on the glial cell populations (figures S5 and S6). Thus, the expression of GFAP and Iba1 (histogram figure 3f) are compared only when the bleeding level was the lowest (\#482 and \#479 for coated and bare electrodes). Although, data are also provided for the other groups (table figure 3f)The density of glial cell is significantly higher than the number neurons at the recording sites that explain the low number of operating sensors (figure 3d). Similar results were observed for the functionalized probes, the proliferation of glial cells being slightly reduced around the probes coated with the multilayers, especially for microglias (figure 3f). Interestingly, this slight improvement is accompanied by a slight increased of the number of operating sensors. These results further demonstrate the biocompatibility of the HA/PLL multilayer films in term of chemical and mechanical inertness. The electrical and histological analyses provide evidences that hyaluronic acid based films do not depress the responses of the sensors and the brain cells. The observations with the functionalized probes are at least similar to those obtained with the control (uncoated) probes, and even slightly improved in term of number of : (1) successful experiments (3/3 versus 1/3), (2) amount of operational sensors (twice higher) and (3) proliferation of glial cells (slightly reduced).

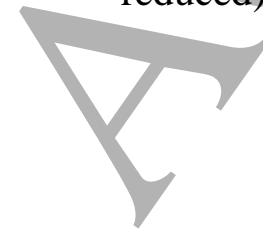


Compatibility of HA coating and graphene bioelectronics. Among all materials used in neuroprostheses, the improvements gained by graphene are clearly significant on the neurons adhesion and outgrowth. ${ }^{10-12}$ For instance, graphene allows neurons to attach and grow without any adhesive coating such as poly-L-lysine (figure S7). Additionally multilayer films can introduce significant improvements for in-vitro cell cultures for instance (figure 2 and S1), and for protecting the monolayer from degradation or delamination during in-vivo assays. Thus combining these two materials could open new perspectives for improving the biocompatibility and the lifetime of neural interfaces. Moreover, the naturally secreted enzyme hyaluronidase ${ }^{47}$ can dissolve the HA coating and reveal the graphene at a predetermined speed rate, in function of the amount of protecting layers. Thus, it enables a direct contact restoration between electronics and cells, together with conferring a progressive drug delivery property thanks to a drug encapsulation in the film. This could further favor the healing process at the implantation site which is a current limitation for numerous brain interfaces, such as the intracortical probes.

However, the functionalization could change the device or material features. To assess such possible drawbacks, we have followed the impact of the HA polymer bounding to graphene. One of the crucial issues is to keep the structural integrity of the monolayer graphene, as well as its high neuronal affinity and its global electrical performance such as high conductivity (mobility and square resistance of CVD graphene being typically around $\mu=5000 \mathrm{~cm} 2 \cdot \mathrm{V}-1 \cdot \mathrm{s}-1$ and $\mathrm{R} \square=$ $500 \Omega / \square)$ and device sensitivity (4mS/V for GFETs). ${ }^{16,17}$

Raman spectroscopy and AFM micrographs have been performed on high quality CVD-grown monolayer graphene before and after HA adhesion (figure 4a-e) to assess the possible impact of the functionalization on the structural properties of the monolayer. In addition to the characteristic peaks of graphene ( $\mathrm{G}$ and 2D band peaks), the Raman spectra show additional resonant modes at 
low frequency (899- $950 \mathrm{~cm}^{-1}, 1090 \mathrm{~cm}^{-1}, 1180 \mathrm{~cm}^{-1}, 1410-1460 \mathrm{~cm}^{-1}$ ) which are characteristic of HA polymer (figure 4b). ${ }^{48,49}$ The contributions of carboxyl and methyl groups $\mathrm{CH}, \mathrm{CH}_{3}$ and $\mathrm{COO}^{-}$ $\left(1330 \mathrm{~cm}^{-1}\right.$ and $\left.1370 \mathrm{~cm}^{-1}\right)$ are certainly superimposed with the defects-related D-band peak $(1350$ $\mathrm{cm}^{-1}$ ) of graphene. Most of all, the amplitude of the D-band peak remains low $\left(\mathrm{I}_{\mathrm{D}} / \mathrm{I}_{\mathrm{G}}<0.06\right)$ after the functionalization, revealing the low density of lattice defects ${ }^{50}$ induced by the binding with HA polymers. These results confirm the adhesion of HA onto graphene, and demonstrate that the HA polymer and the coating procedure do not affect the crystalline quality of the graphene monolayer.

The atomic force micrographs performed before (figure $4 \mathrm{c}$ ) and after the polymer deposition (figure 4d-e) further confirm the integrity and the overall high quality of the graphene monolayer previously transferred on glass substrates. The HA monolayer deposited onto graphene appears first granular at the graphene substrate (figure 4d), certainly because of the high graphene hydrophobicity. Consequently, the homogeneity of the coating is significantly enhanced when adding hydrophobic alkyl (C10) chains on the HA polymer backbone to obtain $\alpha$-HA (depicted figure 4a), as shown within figure 4e. Contact angle measurements show the higher hydrophilicity of the graphene substrates coated with the derivative $\alpha$-HA polymers (figure S8). As expected, the hydrophilicity gets higher when the density of the alkyl chains grafted on the HA backbone is increased, from 10 to $20 \%$ (i.e. on average 10 and 20 chains every 100 repeating disaccharide units of HA). The presence of hydrophobic alkyl chains on the HA backbone promotes adsorption of this polymer on hydrophobic graphene monolayer, simultaneously rendering the surface exposed to the liquid or cells more hydrophilic.

As mentioned previously, HA promotes neurons adhesion and outgrowth in culture when combining it with a positively charged layer such as PLL (figure 1). Interestingly, combining HA 
with positively charged graphene monolayer enables also the adhesion of neurons and an efficient neurites spreading (figure 5a), which are crucial maturation stages for culturing primary neurons and for in-vitro assays. Main cell features, such the number of attached neurons, neurites number and length, are comparable on the active (graphene/HA coated glass) and control (PLL coated glass) samples (figure $5 \mathrm{a}$ and $\mathrm{b}$ respectively). We notice a slightly higher expression of Tau proteins labeling the axon (red) on the graphene substrates, suggesting an earlier polarization of the axon and thus an improvement in the maturation process which is as expected for graphene substrates. ${ }^{12,51}$ Here, our observations show that the high neural affinity of the underneath graphene remains despite the functionalization. As mentioned above, combining graphene with (HA/PLL) multilayers enable also neurons adhesion and outgrowth (figure 2). The neurons density and the surface covered by neurites are further enhanced on the bilayers deposited on graphene after 14 days (figure S2), indicating that combining Graphene and (HA/PLL) films support long-term cell regeneration and survival.

Finally, we have investigated the impact of HA polymers on the electronic transport properties of graphene devices, and in particular by measuring the characteristics of graphene field effect transistor (G-FET), as they offer promising alternatives for future neuronal interfaces. ${ }^{16,17}$ Several G-FETs have been fabricated on sapphire substrates (figure 6a-b) and their maximum transconductance $g_{m}=\partial I_{D S} / \partial V_{G}$ was measured before and after the functionalization. This transconductance is a physical parameter that can illustrate the impact of the HA polymers on the device sensitivity $S$ which can be expressed as:

$$
S=\frac{\Delta G}{G}=g_{m} \times \Delta V_{G} \times \frac{1}{V_{D S} \cdot G}
$$


with $G$ the conductance of the graphene transistor channel, $I_{D S}$ and $V_{D S}$ the drain-source current and voltage, and $V_{G}$ the top-gate voltage applied with a quasi-reference Pt-electrode (figure 6a). The dependence of the channel current with the liquid gate voltage $V_{G}$ shows a typical symmetric ambipolar field effect behavior with a charge neutrality point at $V_{D} \sim 0.4 \mathrm{~V}$ (figure $6 \mathrm{c}$ ). The sensitivity $S$ reaches its maximal value at the inflections of the field effect curve where the transconductance is the highest.

While PLL does not affect the G-FETs properties, ${ }^{16,52}$ HA polymer slightly reduces the transconductance and shifts the charge neutrality point $V_{D}$ toward more positive values (red curves, figure 6c), in agreement with the overall negative charge of HA polymer. However, the minimum of conductance remains unchanged, assessing the fact that the HA polymer and the coating procedure did not damage the integrity of the graphene transistor channel.

The reduced transconductance could stem from a lower value of the charge carrier mobility, and also because the hyaluronic acid changes the interfacial capacitance. The capacitance of the graphene/liquid interface $C_{G}$ is not directly measurable. It results from the serial contribution of the geometric (Helmholtz and diffusive) $C_{G e o}$ and the quantum $C_{Q}$ capacitances of graphene, expressed as the following $1 / \mathrm{C}_{\mathrm{G}} \sim 1 / \mathrm{C}_{\mathrm{Geo}}+1 / \mathrm{C}_{\mathrm{Q}}$. For liquid-gated graphene field effect transistor, the quantum capacitance cannot be neglected, and its variation with the gate voltage should be taken into account to extract the charge carrier mobility values from the transfer curve $I_{D S}-V_{L G}{ }^{53}$ Dedicated methods have been previously developed to model liquid gated G-FETs and extract the mobility and capacitance values in this top gating configuration. ${ }^{54,55}$ The conductivity $\sigma$ of liquid gated graphene transistors is function of the Fermi level and the scattering induced by disorder (adsorbed impurities and defects). It can be expressed as: 


$$
\sigma=G \cdot \frac{L}{W}=g_{0} \frac{6 \sqrt{3}}{4 \pi} \frac{a_{0}^{2} \alpha}{n_{i}}\left|V_{L G}-V_{D}\right| \ln ^{2}\left(\sqrt{\alpha \pi\left|V_{L G}-V_{D}\right|} r\right)
$$

with $g_{0}(0.039 \mathrm{mS})$ the quantum of conductance, $a_{0}(1.42 \AA)$ the $\mathrm{C}-\mathrm{C}$ lattice bond length in graphene, and $r \sim a_{0}$ the short range potential induced by defects scattering, the fitting parameter being $\alpha\left(=\mathrm{n} / \mathrm{V}_{\mathrm{LG}}\right)$ a model interfacial capacitance, and $n_{i}$ the density of impurity or defect.

Figures 5d and 5e compare the fitting curves before and after HA deposition respectively. Adding HA results in a slight increase of defect density $n_{i}$, from 0.5 to $0.66 \times 10^{12} \mathrm{~cm}^{-2}$, while the total interfacial capacitance $C_{L G}=\alpha \times e$ is decreasing, from 0.56 to $0.53 \mu \mathrm{F} . \mathrm{cm}^{-2}$ with and without HA respectively. The total interfacial capacitance includes the serial contribution of the electrical double layer at the interface with the Pt reference electrode, the HA capacitance and the second electrical double layer capacitance at the interface with graphene (figure 6a).

From those values, we can estimate the carriers mobility in the hole and electron regimes, $\mu_{h, e}=$ $L \times g_{m} /\left(W \times V_{D S} \times C_{L G}\right)$, with $g_{m}$ the maximum transconductance for holes and electrons extracted from the transfer curve $\partial I_{D S} / \partial V_{G}\left(V_{G}\right)$ at the maximum inflection points. On bare GFETs (before HA coating), hole and electron mobilities show slightly asymmetric values, being higher in the hole conduction regime $\mu_{\mathrm{h}}=1881 \mathrm{~cm}^{2} \cdot \mathrm{V}^{-1} \cdot \mathrm{s}^{-1}$, while only reaching $\mu_{\mathrm{e}}=1631 \mathrm{~cm}^{2} . \mathrm{V}^{-}$ ${ }^{1} . \mathrm{s}^{-1}$ in the electron conduction regime. These values are consistent with previous studies. ${ }^{54}$ After HA deposition the mobility is slightly reduced for the both (hole and electron) conduction regimes, being almost the same $\mu_{\mathrm{h}, \mathrm{e}}=1283-1296 \mathrm{~cm}^{2} \cdot \mathrm{V}^{-1} \cdot \mathrm{s}^{-1}$ for holes and electrons respectively.

The shift of the charge neutrality voltage (noted $\Delta V_{D}$ in the following) toward more positive values indicates an increase of the hole carrier density. This is consistent with the negative charges doping brought by HA polymers. This variation of charge - expressed as $\Delta \mathrm{n}_{\mathrm{h}}=\Delta \mathrm{V}_{\mathrm{D}} \times \mathrm{c}_{\mathrm{i}} / \mathrm{e}$ with $\mathrm{c}_{\mathrm{i}}$ the 
interfacial capacitance - is about $\Delta \mathrm{n}_{\mathrm{h}}=3.10^{6}$ (with $\mathrm{c}_{\mathrm{i}}=1.06 \mathrm{pF}$, and $\Delta \mathrm{V}_{\mathrm{D}}=0.46 \mathrm{mV}$ ). At the charge neutrality point $V_{D}$, this value should be almost opposite and equal to the positive charges carried by the HA polymers over the transistor channel $\Delta \mathrm{n}_{\mathrm{h}}=-\Delta \mathrm{n}_{\mathrm{HA}}$. Because this value can be estimated and monitored in-situ, it provides an interesting way to follow the integrity of the HA coating when interfacing neurons.

Indeed, the total amount of charge carried by HA is given by (1) the number of HA molecules over the G-FET channel, and (2) the number of charges that a molecule can exchange with the graphene layer. The number of HA molecules depends on the polar surface coverage $P_{S C}$, i.e. the projected surface of $\mathrm{HA}$ molecule on the surface, as the following: $\mathrm{N}_{\mathrm{HA}}=\mathrm{L} \times \mathrm{W} / \mathrm{PSC}_{\mathrm{HA}}$, with $\mathrm{L}$ and $\mathrm{W}$ the length and width of the G-FET channel. Hyaluronic acid being constituted by D-Glucuronic acid and $\mathrm{N}$-acetyl-D-Glucosamine chains for which $\mathrm{Psc}_{\mathrm{SC}}=1.25 \mathrm{~nm}^{2},{ }^{56}$ a full covering should provide almost $1.7 \times 10^{8}$ molecules over the G-FET channel. Finally, the number of charges carried by the $N_{H A}$ molecules can be estimated as the following $\Delta n_{H A}=a \times M_{k} \times N_{H A}$, with $M_{k}$ the Mulliken charge for an absorbed molecule, i.e. the amount of charge that a molecule can exchange with a surface, and $a$ a variable parameter taking into account the availability of charge in a dipole. Considering $\mathrm{M}_{\mathrm{k}}=0.65 \mathrm{e}^{-}$for $\mathrm{HA},{ }^{57}$ and $\mathrm{a}=4 \%$ for grafted dipole on carbon latice, ${ }^{58}$ we found an amount of charge carried by the HA coating of about $\Delta n_{H A}=4.10^{6} \mathrm{e}^{-}$. This value matches well the excess number of holes $\left(\Delta \mathrm{n}_{\mathrm{HA}} \sim-\Delta \mathrm{n}_{\mathrm{h}}\right)$ within the graphene channel. This result shows that monitoring the shift of the chárge neutrality voltage $\Delta V_{D}$ could indeed be used to monitor in-situ the adhesion and degradation in time of HA coatings to restore a direct contact with cells.

At the end, we show the overall homogeneous coating of HA/PLL multilayer films onto graphene samples, similar to the films previously investigated in-vitro and in-vivo on intracortical probe 
(figure 1, 2 and 3 respectively). Graphene substrates (monolayer graphene previously transferred on glass coverslips) were coated with one and five HA/PLL bilayers. Fluorescein isothiocyanate FITC grafted PLL (FITC-PLL) was used as the top layer in both cases to assess for the bilayers deposition. Figure 7 shows the frontier between the graphene and the graphene-free glass substrate. As expected, the fluorescence intensity is higher above the graphene-free area. ${ }^{9}$ The ratio of fluorescence intensity over these two areas without and with graphene is about $\mathrm{I}_{\mathrm{wo}} / \mathrm{I}_{\mathrm{w}}=2.40$ when coating the substrate with one HA/PLL bilayer (figure $7 \mathrm{a}$ ), and is reduced twice $\left(\mathrm{I}_{\mathrm{wo}} / \mathrm{I}_{\mathrm{w}}=1.24\right)$ when increasing the number of bilayer up to five (figure $7 \mathrm{~b}$ ). The reduced quenching effect observed for five bilayers is related to the higher distance of FITC-PLL from the graphene substrate, confirming the higher thickness of HA/PLL deposited film. Interestingly, this multilayer coating exhibit ripples at the graphene/glass border (close-up view figure 7b), suggesting possible edge effects, or mechanical strains either within the multilayer film or within the graphene monolayer. These results demonstrate the overall homogeneity of the HA/PLL multilayer film and the ability to implement it on large graphene monolayer.

In perspective to this study, the controlled enzymatic degradation of HA coatings should be investigated to restore progressively a direct contact with the cells at the recording sites, and still protect the graphene monolayer during its insertion through the brain tissues and deep cortical layers. Moreover, the type and dynamics of synapses that are overexpressed on the bilayers film could be further assessed, as well as the possible impact of the coatings on the electrical activity of the network either by using conventional patch-clamp technique or the GFET array.

Conclusion. All together these results show that HA polymeric films could be implemented on intracortical probes and graphene substrates, without altering the material and device features, as well as the neurons and tissues response, either in culture or within intracortical brain layers. These 
results validate the interest of using HA derivative polymers to coat bioelectronic probes based on graphene, as it could provide many additional advantages to expand the lifetime and reliability of brain implants. Moreover HA provides a robust basement for further development of biomaterials such as natural hydrogels, to add new functionalities for drugs delivery, growth factors and stem cell scaffolds to counterpart the glial scar formation. The demonstrated possibility to combine HA coatings with graphene bioelectronics provides exciting solutions to prevent possible delamination of graphene monolayers in-vivo, and to trigger the healing process whichis the key for a stable and efficient electrical coupling to neurons. In that way, such a protective biomimetic shell could be helpful for improving the acceptance of a wide range of materials used in neurotechnology.

\section{FIGURES}

Figure 1. In-vitro assays DIV5. (a) Immuno-fluorescent micrographs of neurons, cultured 5 days on glass coverslip coated with poly-L-lysine (control sample) and five HA/PLL bilayers, with PLL and HA as the top layer, (HA/PLL) $5 / 5$ and (HA/PLL)5/4 respectively. For each condition, high and low magnification is shown, scale bars are $1000 \mu \mathrm{m}$ and $50 \mu \mathrm{m}$ respectively. Soma, neurites, axons, and synapses are labeled in blue (DAPI), green (YL1/2), gray (Tau) and red (Synapsin). Single channel micrograph for synapsin allows to discriminate the synapses. (b) Statistic analysis compares the neuron density, total area covered by neurites and axons, and the synapse density for each condition. For synapses counting, a high threshold was used to detect bright puncta around the soma, and a low threshold was used to detect puncta along neurites. (significant level *** $\mathrm{p}<$ 0.001, total number of counted cells: 3785, 4204 and 3253 for PLL, (HA/PLL)5/5 and (HA/PLL)5/4 respectively).

Figure 2. In-vitro assays DIV14. Immuno-fluorescent micrographs of neurons at DIV14, cultured on PLL-coated glass, and on five HA/PLL bilayers deposited on glass and on monolayer graphene. For each condi-tion, neurons are stained with DAPI, YL1/2 and synapsin to label the soma, the neurite and the synapses. Scale bars are $50 \mu \mathrm{m}$. For the bilayers deposited on graphene and glass, 
the neurons are widely distributed across the thickness of the multilayers. Multiple regions were sub-sampled across z-space for the (HA/PLL) bilayers deposited on glass (figure S2).

Figure 3. Impact of HA films on intracortical probes. Five HA/PLL bilayers were deposited on commercial tungsten electrodes arrays, implanted in the motor cortex of rats, and compared to control samples (same probes without coating). (a) Fluorescent micrographs of the coated electrodes. FITC-PLL $\left(\lambda_{\text {exc }}=488 \mathrm{~nm}\right)$ was deposited as the top layer for assessing the multilayer deposition. (b) Typical (surimposed) spikes recorded during locomotion. Scale bar $100 \mu \mathrm{V}$ and 1ms. Single spikes are extracted from the voltage time trace shown in (c). Scale bar $100 \mu \mathrm{V}$. (d) Percent of control (black) and coated (orange) electrodes enable to detect single spikes. (e) Postmortem immuno-fluorescent micrographs of neural tissues ( 7 weeks after implantation surgery) showing the astrocytes (GFAP) and microglia (IbA1) around the coated (active) and bare (control) electrodes, on the left and right column respectively. Scale bar $2 \mathrm{~mm}$. (f) Corresponding histograms, comparing the total surface covered by astrocytes and microglia in each conditions, i.e. with (active) and without (control) HA/PLL bilayers, when the bleeding level was the lowest (\#482 and \#479 for coated and bare electrodes). Table shows the results obtained for the other groups.

Figure 4. HA adhesion onto monolayer graphene. (a) Schematics of the biopolymer customization for a homogeneous adhesion on hydrophobic graphene monolayers. The HA backbone is functionalized with alkylated hydrophobic $\left(\mathrm{CH}_{3}\right)$-chains promoting polymer attachment onto the hydrophobic substrate. (b) Typical Raman spectrum acquired on HA-coated graphene samples. (c-e) Atomic force micrographs of typical bare graphene monolayer (c), then coated with a single layer of (d) HA and (e) HA modified with hydrophobic chains $\alpha-H A$. Bottom : z-profiles of the two coatings, left: HA and right: $\alpha$-HA, measured along the green line in (d) and (e).

Figure 5. Neurons adhesion on HA-coated graphene. Immuno-fluorescent micrographs of primary hippocampal neurons cultured on glass coverslips covered with graphene and HA monolayers (a), and coated with a PLL (b) and HA (c) single layer (control samples). The soma, neurites, and axon are labeled in blue (DAPI), green (YL1/2), and red (Tau). 1 Day-in-vitro. 
Figure 6. Impact of HA on the electronic properties of G-FETs. (a) Schematics of the functionalized graphene field effect transistors G-FETs. The electrical equivalent circuit illustrates the coupling between the liquid gate and the graphene FET channel. Optical micrographs of the G-FET array before (zoomed view) and after the passivation of the Ti/Au metallic leads. The graphene FET channels are $10 \times 20 \mu \mathrm{m}$ long and wide. (c) The G-FET conductance - before (gray) and after (red) HA deposition - is recorded as a function of the liquid gate voltage $V_{G}$ applied with a quasi-reference Pt electrode. Each curve corresponds to a different device. Thicker curves are the mean average value over all tested devices. The average curves are fitted to extract the charge carriers density and mobility, before (d) and after (e) functionalization (see text for fitting parameters).

Figure 7. Adhesion of HA/PLL multilayer film on graphene monolayer. Fluorescent optical micrographs of one (a) and five (b) HA/PLL bilayers deposited on monolayer graphene previously transferred on glass coverslips. FITC-PLL $\left(\lambda_{\text {ex́c }}=488 \mathrm{~nm}\right)$ was used for deposition of the last layer. Exposure time is $1500 \mathrm{~ms}$ (a), and $500 \mathrm{~ms}$ (b). Inset: zoomed view of the frontier with graphene. 


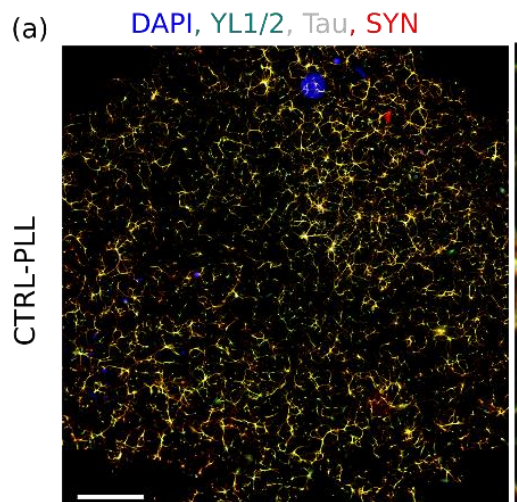

DAPI, YL1/2, Tau, SYN
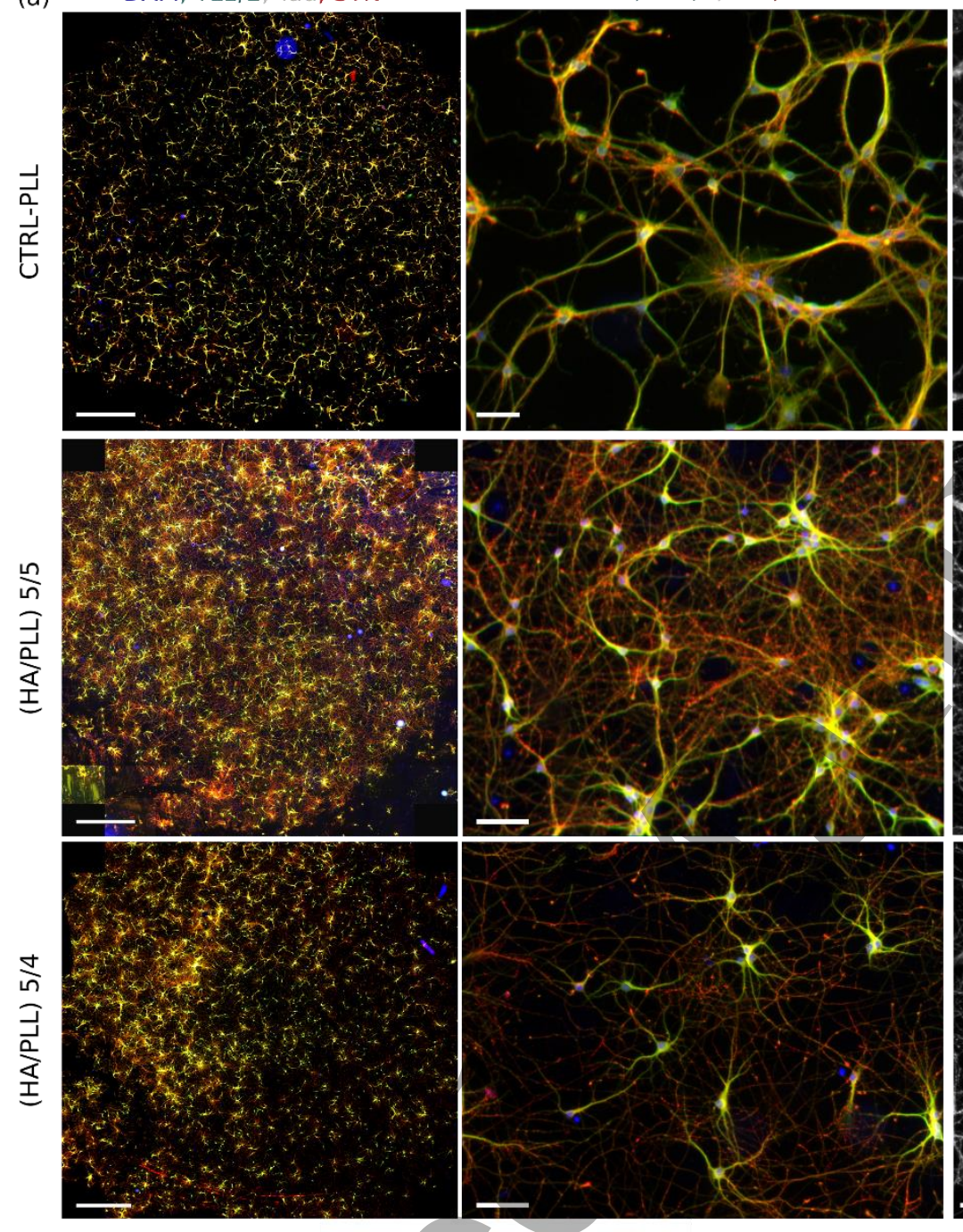

(b)
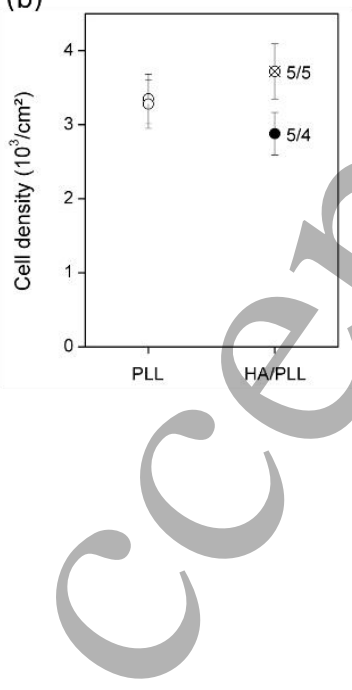

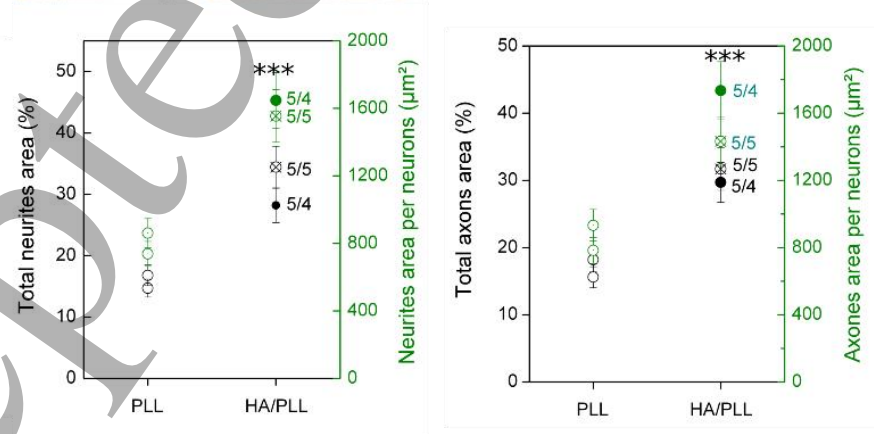

Figure 1.

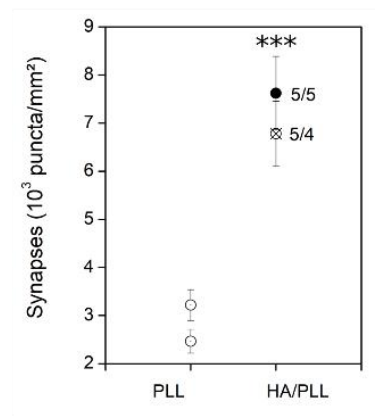




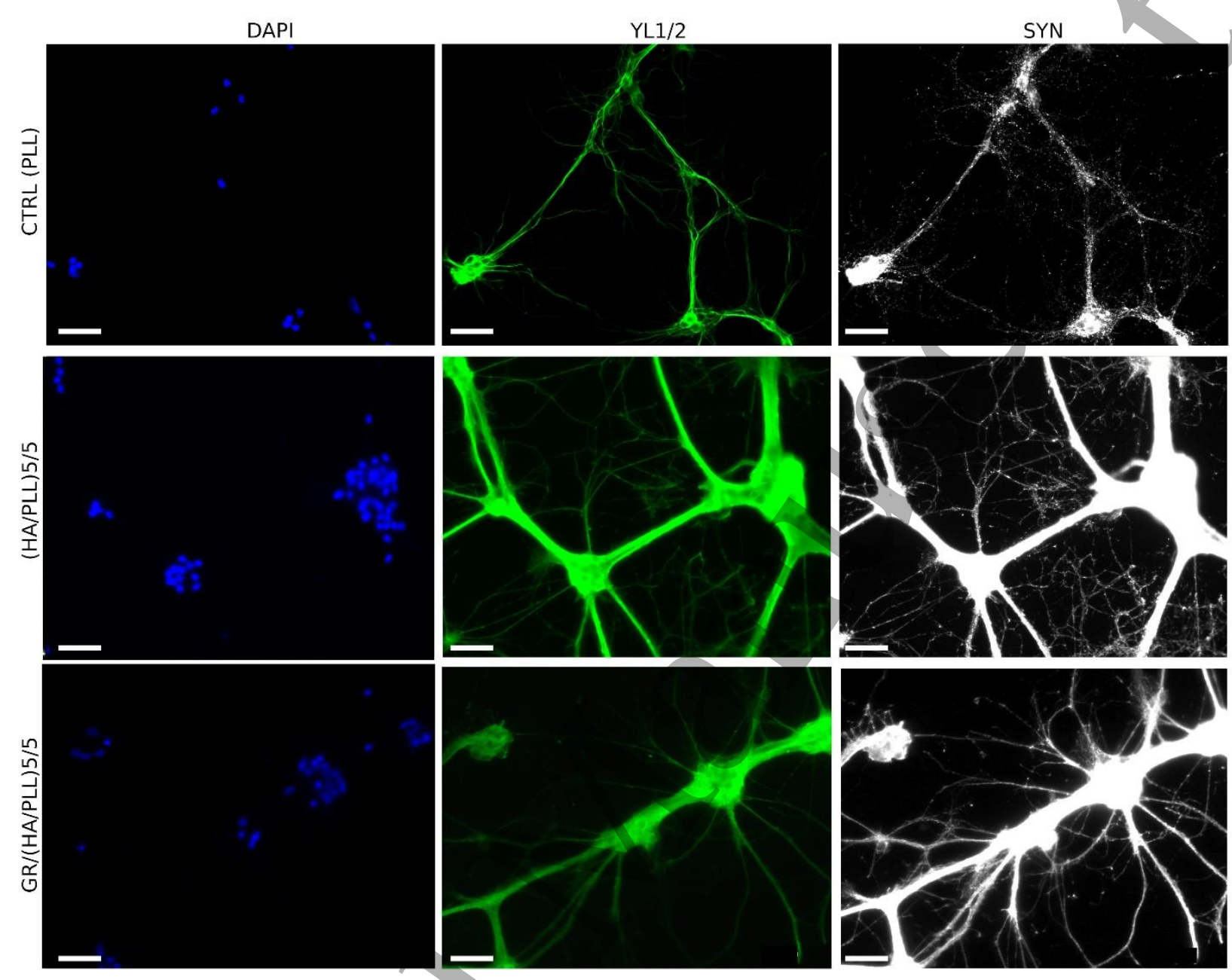

figure 2 . 

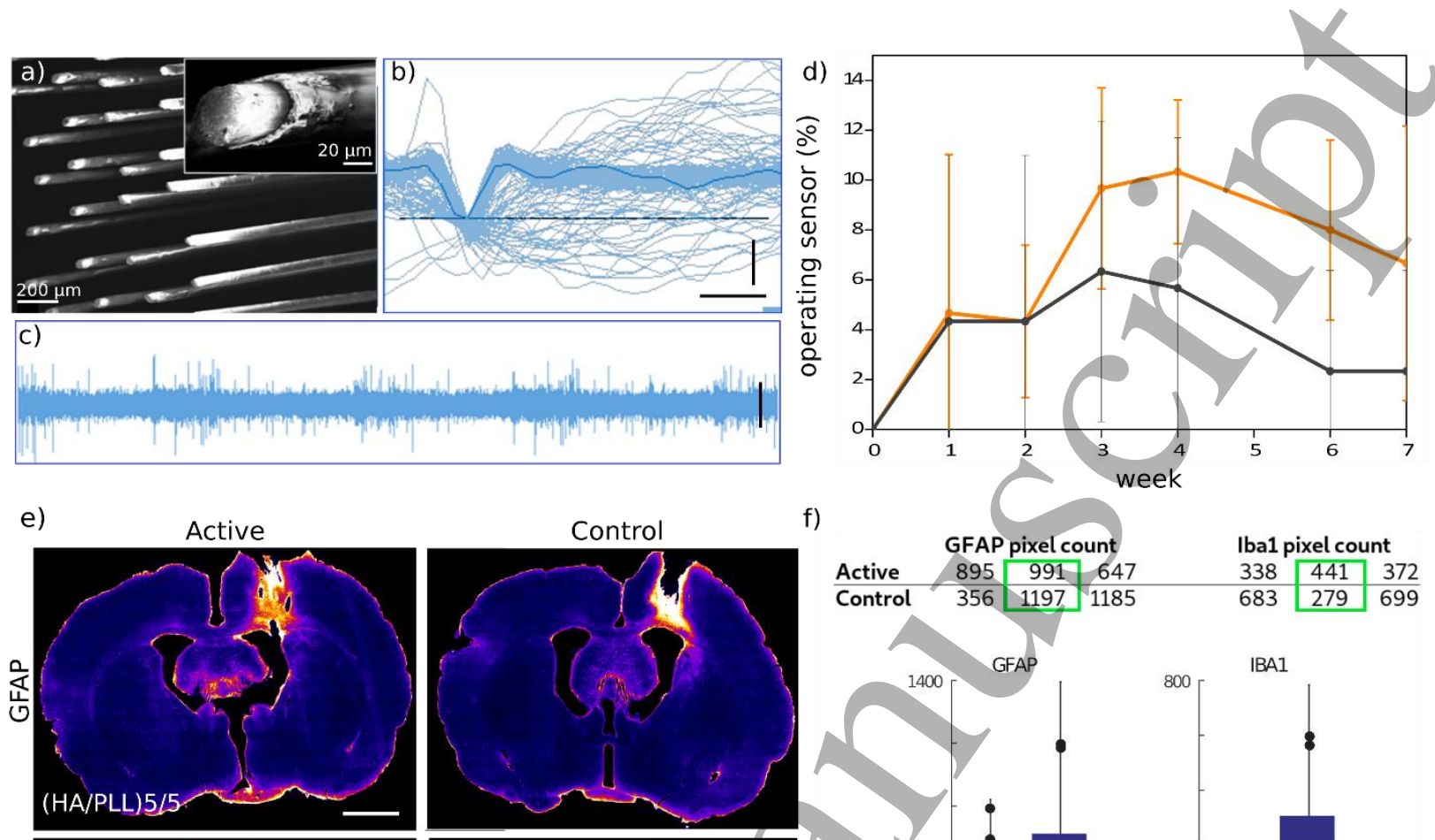

f)
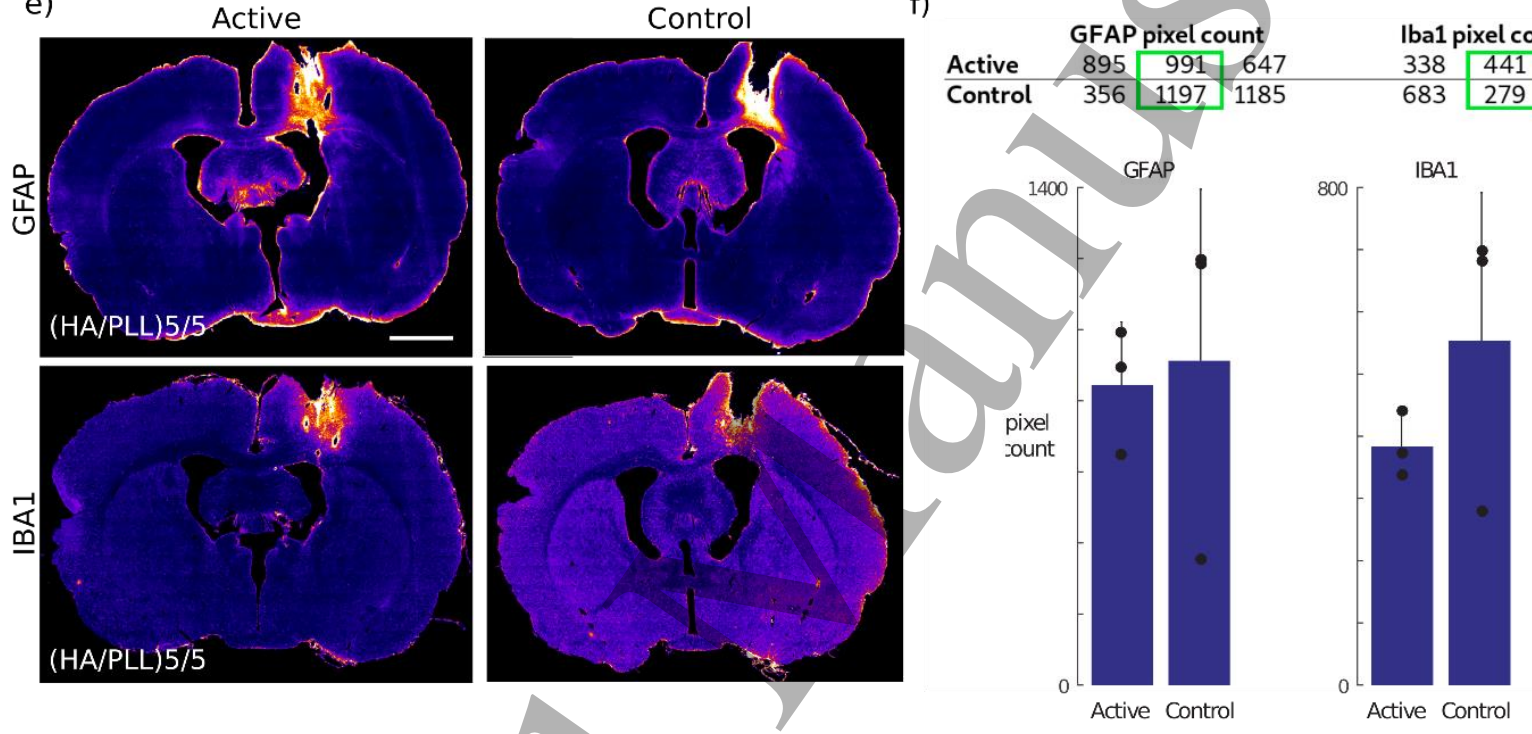

Figure 3. 
a)

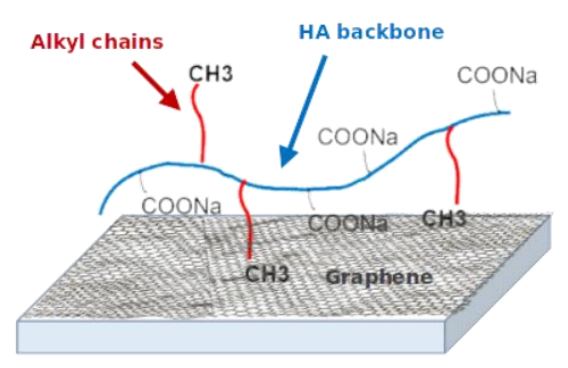

d) With HA

b)

c) Bare Graphene
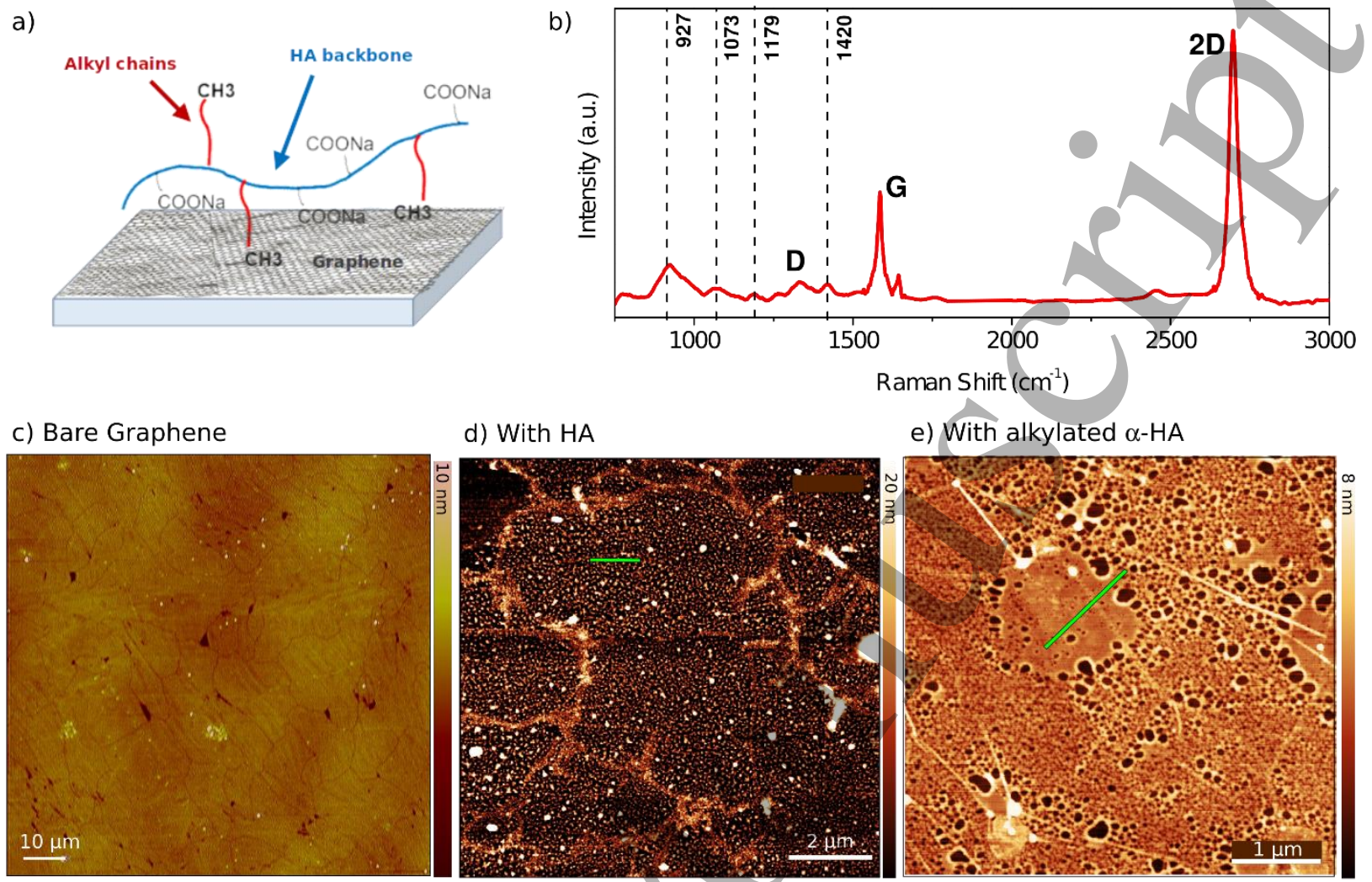

e) With alkylated $\alpha-H A$
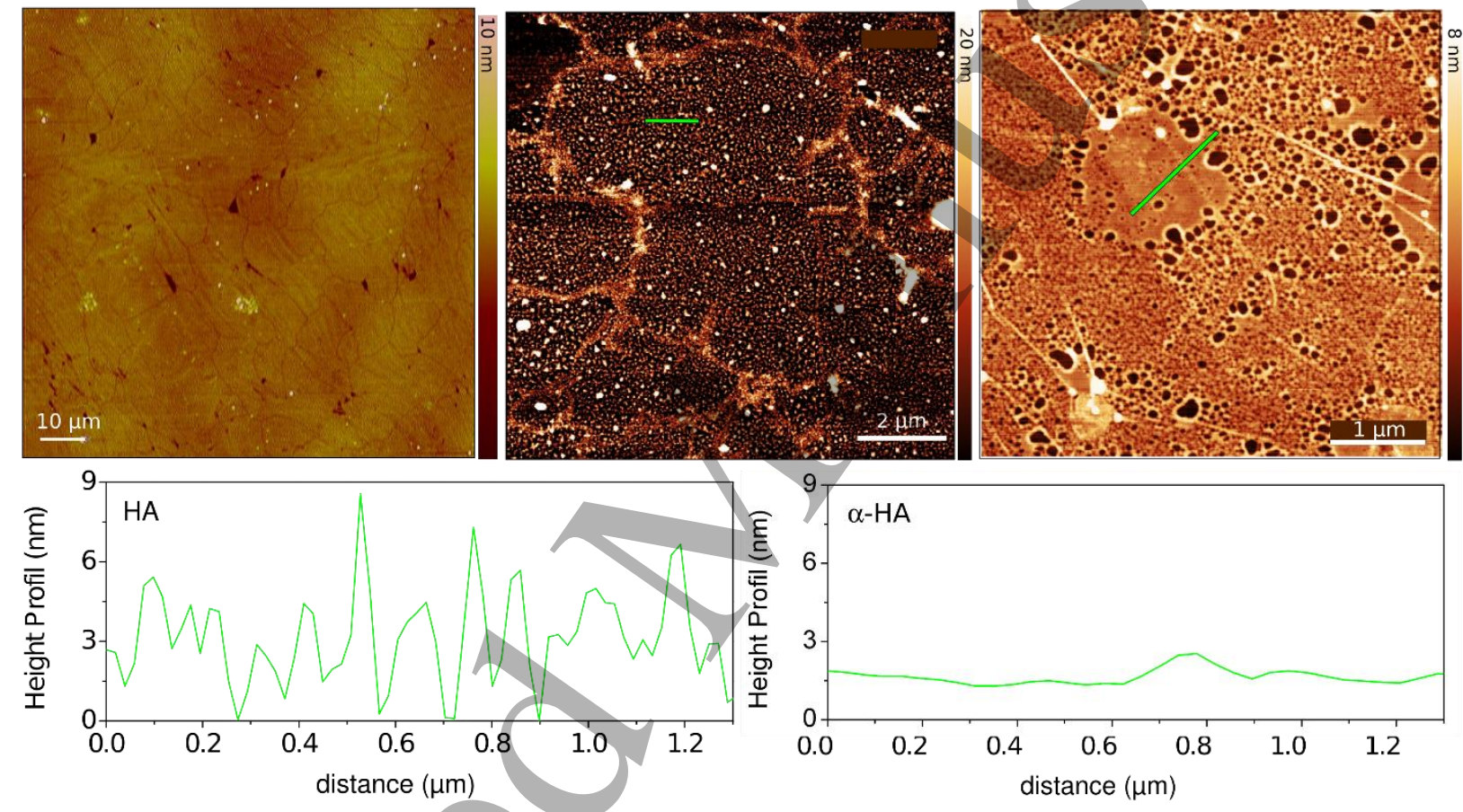

Figure 4. 

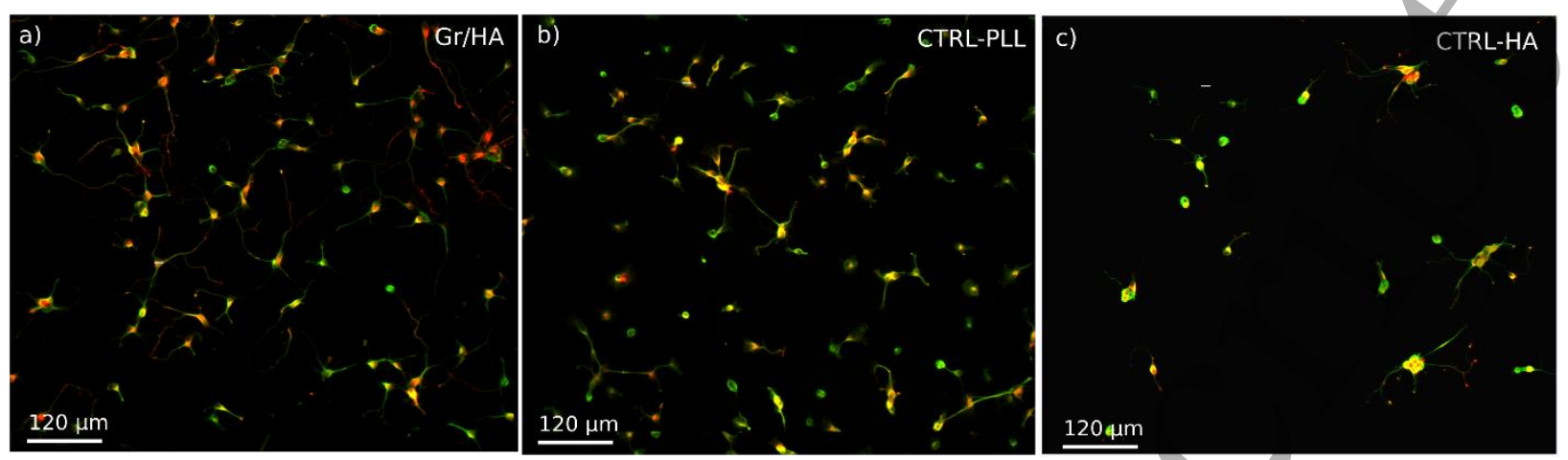

Figure 5.
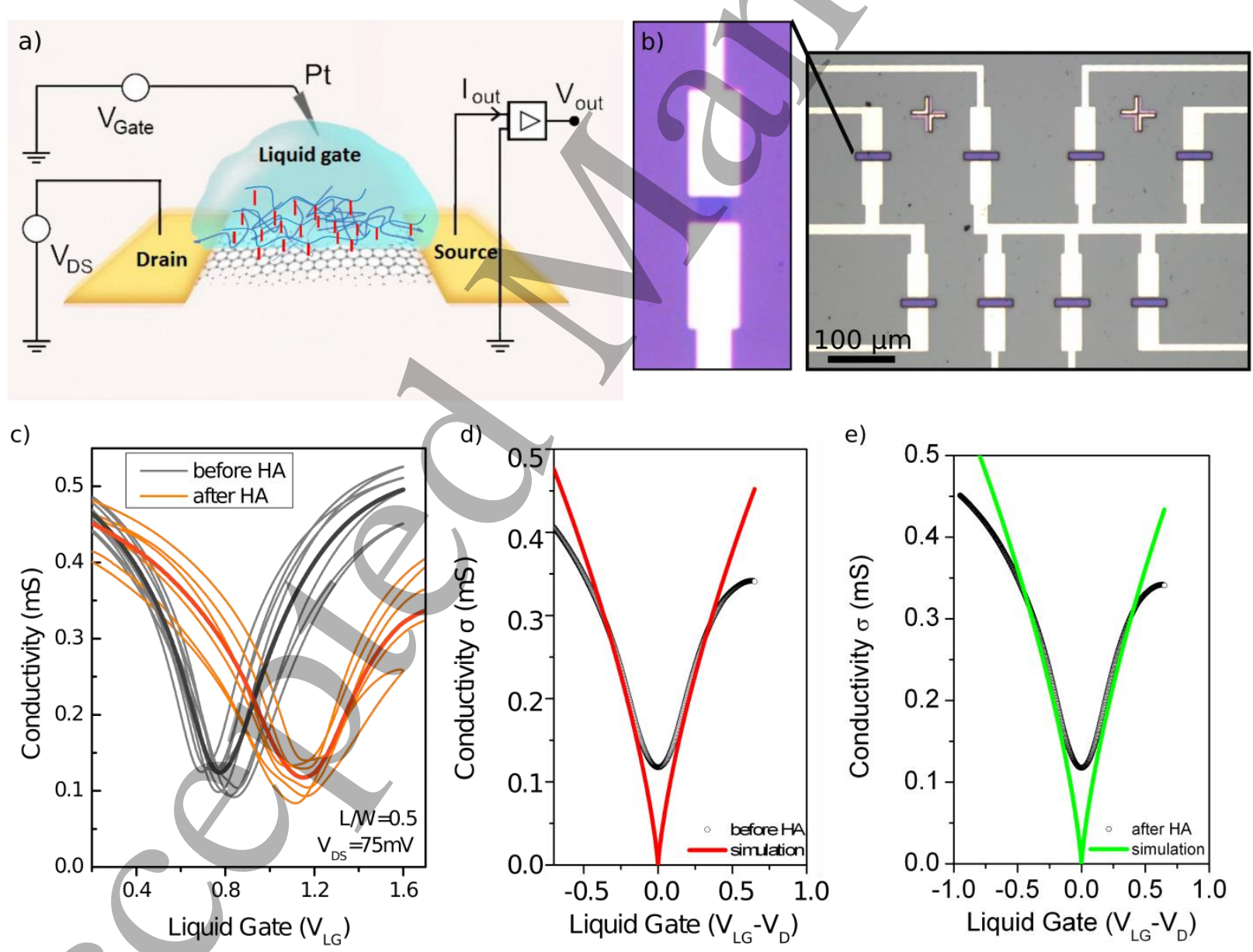

Figure 6. 

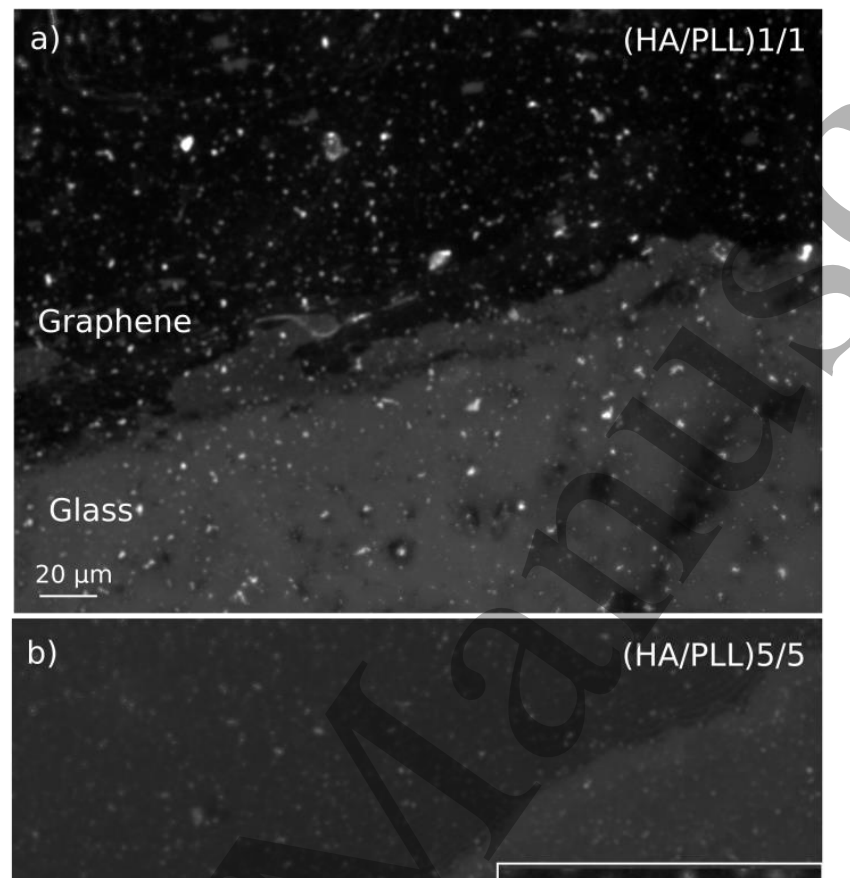

Graphene

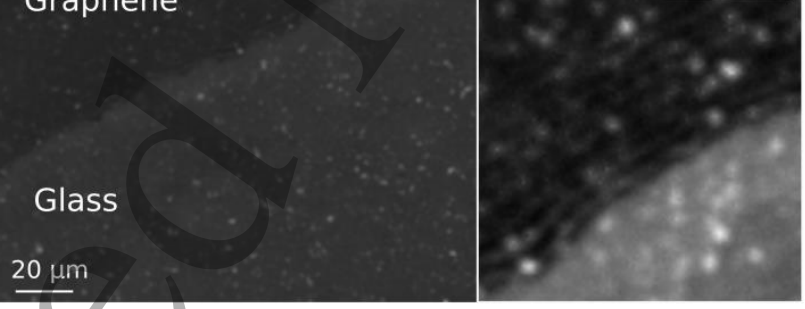

Figure 7. 


\section{ASSOCIATED CONTENT}

Supporting Information. Supplementary figures S1-S7.

Supplementary video 1. Freely-moving recordings. This video shows freely-moving implanted animal during the recordings (26 experiments per probe and per week).

\section{AUTHOR INFORMATION}

Corresponding Author

*E-mail: cecile.delacour@neel.cnrs.fr

\section{Author Contributions}

The manuscript was written through contributions of all authors. All authors have given approval to the final version of the manuscript.

\section{Notes}

The authors declare no competing financial interest.

\section{ACKNOWLEDGMENTS}

The authors thank the help provided by nanofabrication (Nanofab) and biotechnology (Biofab) facilities at Neel Institute. This work is partially supported by la Région Rhône-Alpes, and by the french Agence Nationale de la Recherche, under the projects ANR-10-LABX-51-01 (Labex LANEF du Programme d'Investissements d'Avenir) the Lab Alliances on Nanosciences - Energies for the Future, and ANR-18-CE42-0003-01 NANOMESH.

\section{ABBREVIATIONS}


HA Hyaluronic Acid, PLL Poly-L-Lysine, G-FET Graphene Field Effect Transistor, LbL Layerby-layer, PDMS Polydimethylsiloxane, CVD Chemical Vapor Deposition.

\section{REFERENCES}

1. Jun, J. J. et al. (2017). Fully integrated silicon probes for high-density recording of neural activity. Nature, 551(7679), 232.

2. Kim, G. et al. (2018). Recent Progress on Microelectrodes in Neural Interfaces. Materials, 11(10), 1995.

3. Hochberg, L. R. et al. (2012). Reach and grasp by people with tetraplegia using a neurally controlled robotic arm. Nature, 485(7398), 372.

4. Asboth, L., et al. (2018). Cortico-reticulo-spinal circuit reorganization enables functional recovery after severe spinal cord contusion. Nature neuroscience, 21(4), 576.

5. Chen, R. Canales, A., and Anikeeva, P. (2017). Neural recording and modulation technologies. Nature Reviews Materials, 2(2), 16093.

6. Polikov VS, Tresco PA, Reichert WM. (2005) Response of brain tissue to chronically implanted neural electrodes. Journal of neuroscience methods, 148, 1-18

7. Lacour, S. P., Courtine, G., Guck, J. (2016). Materials and technologies for soft implantable neuroprostheses. Nature Reviews Materials, 1, 16063

8. Barrese, J. C., Rao, N., Paroo, K., Triebwasser, C., Vargas-Irwin, C., Franquemont, L., and Donoghue, J. P. (2013). Failure mode analysis of silicon-based intracortical microelectrode arrays in non-human primates. Journal of neural engineering, 10(6), 066014 
9. Barrese, J. C., Aceros, J. and Donoghue, J. P. (2016) Scanning electron microscopy of chronically implanted intracortical microelectrode arrays in non-human primates. J. Neural Engineer. 13, 026003

10. Park, S. Y., Park, J., Sim, S. H., Sung, M. G., Kim, K. S., Hong, B. H., Hong, S. (2011). Enhanced differentiation of human neural stem cells into neurons on graphene. Advanced Materials, 23(36)

11. Li, N., Zhang, X., Song, Q., Su, R., Zhang, Q., Kong, T., Liu, L., Jin, G., Tang, M. and Cheng, G. (2011). The promotion of neurite sprouting and outgrowth of mouse hippocampal cells in culture by graphene substrates. Biomaterials, 32(35), 9374

12. Veliev, F., Briançon-Marjollet, A., Bouchiat, V., and Delacour, C. (2016). Impact of crystalline quality on neuronal affinity of pristine graphene. Biomaterials, 86,33

13. Lee, C., Wei, X., Kysar, J. W., Hone, J. (2008). Measurement of the elastic properties and intrinsic strength of monolayer graphene. science, 321(5887), 385

14. S. Chen, L. Brown, M. Levendorf, W. Cai, S.-Y. Ju, J. Edgeworth, X. Li, C.W. Magnuson, A. Velamakanni, R.D. Piner, J. Kang, J. Park, R.S. Ruoff (2011). Oxidation resistance of graphene-coated $\mathrm{Cu}$ and $\mathrm{Cu} / \mathrm{Ni}$ alloy, ACS Nano 5,1321

15. D. Prasai, J.C. Tuberquia, R.R. Harl, G.K. Jennings, B.R. Rogers, K.I. Bolotin (2012). Graphene: corrosion-inhibiting coating, ACS Nano 6, 1102 
16. Veliev, F., Han, Z., Kalita, D., Briançon-Marjollet, A., Bouchiat, V., and Delacour, C. (2017). Recording spikes activity in cultured hippocampal neurons using flexible or transparent graphene transistors. Frontiers in Neuroscience, 11, 466

17. Blaschke, B. M., Tort-Colet, N., Guimerà-Brunet, A., Weinert, J., Rousseau, L., Heimann, A., Drieschner, S., Kempski, O., Villa, R., Sanchez-Vives, M.V., and Garrido, J. A. (2017). Mapping brain activity with flexible graphene micro-transistors. 2D Materials, 4(2), 025040

18. Girish, C. M., Sasidharan, A., Gowd, G. S., Nair, S., Koyakutty, M. (2013). Confocal Raman imaging study showing macrophage mediated biodegradation of graphene in vivo. Advanced healthcare materials, 2(11), 1489

19. Kotchey, G. P., Allen, B. L., Vedala, H., Yanamala, N., Kapralov, A. A., Tyurina, Y. Y., Klein-Seetharaman, J., Kagan, V.E., Star, A. (2011). The enzymatic oxidation of graphene oxide. ACS nano, 5(3), 2098

20. Kurapati, R., Russier, J., Squillaci, M. A., Treossi, E., Ménard-Moyon, C., Rio-Castillo, D., Vazquez, E., Samori, P., Palermo, V., Bianco, A. (2015). Dispersibility-Dependent Biodegradation of Graphene Oxide by Myeloperoxidase. Small, 11(32), 3985

21. Minev, I. R., Musienko, P., Hirsch, A., Barraud, Q., Wenger, N., Moraud, E. M., Gandar, J., Capogrosso, M., Milekovic, T., Asboth, L., Torres, R.F., Vachicouras, N., Liu, Q., Pavlova, N., Duis, S., Larmagnac, A., Vörös, J., Micera, S., Suo, Z., Courtine, G., Lacour, S.P. (2015). Electronic dura mater for long-term multimodal neural interfaces. Science, $347(6218), 159$ 
22. Burdick, J. A., Prestwich, G. D. (2011). Hyaluronic acid hydrogels for biomedical applications. Advanced materials, 23(12).

23. Highley, C. B., Prestwich, G. D., Burdick, J. A. (2016). Recent advances in hyaluronic acid hydrogels for biomedical applications. Current opinion in biotechnology, 40, 35

24. Tarus, D., Hamard, L., Caraguel, F., Wion, D., Szarpak-Jankowska, A., van der Sanden, B., Auzély-Velty, R. (2016). Design of hyaluronic acid hydrogels to promote neurite outgrowth in three dimensions. ACS applied materials \& interfaces, 8(38), 25051

25. Knopf-Marques, H., Pravda, M., Wolfova, L., Velebny, V., Schaaf, P., Vrana, N. E., Lavalle, P. (2016). Hyaluronic acid and its derivatives in coating and delivery systems: applications in tissue engineering, regenerative medicine and immunomodulation. Advanced healthcare materials, 5(22), 2841

26. Lee, J. Y., Khaing, Z. Z., Siegel, J. J., Schmidt, C. E. (2015). Surface modification of neural electrodes with a pyrrole-hyaluronic acid conjugate to attenuate reactive astrogliosis in vivo. RSC Advances, 5(49), 39228

27. Sridar, S., Churchward, M. A., Mushahwar, V. K., Todd, K. G., Elias, A. L. (2017). Peptide modification of polyimide-insulated microwires: Towards improved biocompatibility through reduced glial scarring. Acta Biomaterialia, 60, 154

28. Grand, L., Wittner, L., Herwik, S., Göthelid, E., Ruther, P., Oscarsson, S., Neves, H., Dombovari, B., Csercsa, R., Karmos, G., Ulbert, I. (2010). Short and long term biocompatibility of NeuroProbes silicon probes. Journal of neuroscience methods, 189(2), 216 
29. Kadi, S., Cui, D., Bayma, E., Boudou, T., Nicolas, C., Glinel, K., Picart, C., Auzély-Velty, R. (2009). Alkylamino hydrazide derivatives of hyaluronic acid: synthesis, characterization in semidilute aqueous solutions, and assembly into thin multilayer films. Biomacromolecules, 10(10), 2875

30. Schneider, C.A., Rasband, W.S., Eliceiri, K.W. (2012). NIH Image to ImageJ: 25 years of image analysis. Nat Methods 9,671

31. DiGiovanna, J., Dominici, N., Friedli, L., Rigosa, J., Duis, S., Kreider, J., Beauparlant, J., van den Brand, R., Schieppati, M., Micera, S., and Courtine, G. (2016). Engagement of the Rat Hindlimb Motor Cortex across Natural Locomotor Behaviors. Journal of Neuroscience, 36(40), 10440

32. van den Brand (2012). Restoring voluntary control of locomotion after paralyzing spinal cord injury. science, 336(6085), 1182-1185.

33. Z. Han, A. Kimouche, D. Kalita, A. Allain, H. Arjmandi-Tash, A. Reserbat-Plantey, L. Marty, S. Pairis, V. Reita, N. Bendiab, J. Coraux, V. Bouchiat (2014). Homogeneous optical and electronic properties of graphene due to the suppression of multilayer patches during CVD on copper foils, Adv. Funct. Mater. 24, 964-970.

34. Szarpak, A., Cui, D., Dubreuil, F., De Geest, B. G., De Cock, L. J., Picart, C., and AuzélyVelty, R. (2010). Designing hyaluronic acid-based layer-by-layer capsules as a carrier for intracellular drug delivery. Biomacromolecules, 11(3), 713-720.

35. Xu, L. P., Meng, J., Zhang, S., Ma, X., and Wang, S. (2016). Amplified effect of surface charge on cell adhesion by nanostructures. Nanoscale, 8(25), 12540-12543. 
36. Mattson, M. P., Haddon, R. C., and Rao, A. M. (2000). Molecular functionalization of carbon nanotubes and use as substrates for neuronal growth. Journal of Molecular Neuroscience, 14(3), 175-182.

37. Hu, M., Sabelman, E. E., Tsai, C., Tan, J., and Hentz, V. R. (2000). Improvement of Schwann cell attachment and proliferation on modified hyaluronic acid strands by polylysine. Tissue engineering, 6(6), 585-593.

38. Yue, Z., Liu, X., Molino, P. J., and Wallace, G. G. (2011). Bio-functionalisation of polydimethylsiloxane with hyaluronic acid and hyaluronic acid-collagen conjugate for neural interfacing. Biomaterials, 32(21), 4714-4724.

39. Kreeger, P. K., Strong, L. E., and Masters, K. S. (2018). Engineering Approaches to Study Cellular Decision Making. Annual review of biomedical engineering, 20, 49-72.

40. Schneider, A., Francius, G., Obeid, R., Schwinté, P., Hemmerlé, J., Frisch, B., Schaaf, P., Voegel J.C., Senger, B., and Picart, C. (2006). Polyelectrolyte multilayers with a tunable Young's modulus: influence of film stiffness on cell adhesion. Langmuir, 22(3), 1193

41. Im, C., and Seo, J. M. (2016). A review of electrodes for the electrical brain signal recording. Biomedical Engineering Letters, 6(3), 104-112.

42. Lacour, S.P., Benmerah, S., Tarte, E., FitzGerald, J., Serra, J., McMahon, S., Fawcett, J., Graudejus, O., Yu, Z. and Morrison, B. (2010). Flexible and stretchable micro-electrodes for in vitro and in vivo neural interfaces. Medical \& biological engineering \& computing, 48(10), 945-954 
43. Johnston, I. D., McCluskey, D. K., Tan, C. K. L., and Tracey, M. C. (2014). Mechanical characteriza-tion of bulk Sylgard 184 for microfluidics and microengineering. Journal of Micromechanics and Microengineering, 24(3), 035017

44. Rivnay, J., Wang, H., Fenno, L., Deisseroth, K., and Malliaras, G. G. (2017). Nextgeneration probes, particles, and proteins for neural interfacing. Science Advances, 3(6), e1601649

45. Lee, I. C., Wu, Y. C., Cheng, E. M., and Yang, W. T. (2015). Biomimetic niche for neural stem cell differentiation using poly-L-lysine/hyaluronic acid multilayer films. Journal of biomaterials applications, 29(10), 1418

46. Wenger, N., Moraud, E. M., Raspopovic, S., Bonizzato, M., DiGiovanna, J., Musienko, P., Morari, M., Micera, S., and Courtine, G. (2014). Closed-loop neuromodulation of spinal sensorimotor circuits controls refined locomotion after complete spinal cord injury. Science translational medicine, 6(255), $255 \mathrm{ra} 133$

47. Kurisawa, M., Chung, J. E., Yang, Y. Y., Gao, S. J., and Uyama, H. (2005). Injectable biodegradable hydrogels composed of hyaluronic acid-tyramine conjugates for drug delivery and tissue engineering. Chemical communications, (34), 4312

48. Bansil, R., Yannas, I. V., and Stanley, H. E. (1978). Raman spectroscopy: a structural probe of glycosaminoglycans. Biochimica et Biophysica Acta (BBA)-General Subjects, 541(4), 535 
49. Barrett, T. W., and Peticolas, W. L. (1979). Laser Raman inelastic light scattering investigations of hyaluronic acid primary and secondary structure. Journal Of Raman Spectroscopy, 8(1), 35

50. Das, A., Chakraborty, B., and Sood, A. K. (2008). Raman spectroscopy of graphene on different substrates and influence of defects. Bulletin of Materials Science, 31(3), 579

51. Bendali, A., Hess, L. H., Seifert, M., Forster, V., Stephan, A. F., Garrido, J. A., and Picaud, S. (2013). Purified neurons can survive on peptide-free graphene layers. Advanced healthcare materials, 2(7), 929-933.

52. Wang, J., Zhao, Y., Ma, F. X., Wang, K., Wang, F. B., and Xia, X. H. (2013). Synthesis of a hydrophilic poly-L-lysine/graphene hybrid through multiple non-covalent interactions for biosensors. Journal of materials Chemistry B, 1(10), 1406-1413.

53. Xia, J., Chen, F., Li, J., and Tao, N. (2009). Measurement of the quantum capacitance of graphene. Nature nanotechnology, 4(8), 505

54. Ferreira, A., Viana-Gomes, J., Nilsson, J., Mucciolo, E. R., Peres, N. M., and Neto, A. C. (2011). Unified description of the dc conductivity of monolayer and bilayer graphene at finite densities based on resonant scatterers. Physical Review B, 83(16), 165402.

55. Vieira, N. C. S., Borme, J., Machado Jr, G., Cerqueira, F., Freitas, P. P., Zucolotto, V., Peres, NMR. and Alpuim, P. (2016). Graphene field-effect transistor array with integrated electrolytic gates scaled to $200 \mathrm{~mm}$. Journal of Physics: Condensed Matter, 28(8), 085302 
56. Kim, S., Thiessen, P. A., Bolton, E. E., Chen, J., Fu, G., Gindulyte, A., Han, L., He, J., He, S., Shoemaker Jiyao Wang, B.A., Yu, B., Zhang, J., Bryant, S.H. (2015). PubChem substance and compound databases. Nucleic acids research, 44(D1), D1202

57. Adrian-Scotto, M., Ben Abdallah, K., Mallet, G. and Vasilescu, D. (2003).Quantum molecular modeling of free radical saccharides from hyaluronan. J. Mol. Struct. Theochem., 636, 89-113.

58. Zhao, J., Buldum, A., Han, J. and Lu, J.P. (2002). Gas molecule adsorption in carbon nanotubes and nanotube bundles. Nanotechnology, 13(2), 195

59. Chang, H., Tang, L., Wang, Y., Jiang, J., and Li, J. (2010). Graphene fluorescence resonance energy transfer aptasensor for the thrombin detection. Analytical Chemistry, 82(6), 2341 